\title{
AMCoR
}

Asahikawa Medical College Repository http://amcor.asahikawa-med.ac.jp/

Journal of Thrombosis and Thrombolysis (2005. Apr) 19(2):101-103.

Multiple and recurrent systemic thrombotic events associated with congenital anomaly of inferior vena cava

Takehara, Naohumi ; Hasebe, Naoyuki ; Enomoto, Setsu ;

Takeuchi, Toshiharu ; Takahashi, Fumihiko ; Ota,

Takahumi ; Kawamura, Yuichiro ; Kikuchi, Kenjiro 


\section{Multiple and recurrent systemic thrombotic events associated with congenital anomaly of inferior vena cava}

Naohumi Takehara ', Naoyuki Hasebe ', Setsu Enomoto ${ }^{2}$, Toshiharu Takeuchi', Fumihiko Takahashi ${ }^{1}$, Takahumi Ota ${ }^{1}$, Yuichiro Kawamura ${ }^{1}$, Kenjiro Kikuchi ${ }^{1}$

1. The first Department of Medicine, Asahikawa Medical College, Asahikawa, Japan

2. Department of Neurology, Asahikawa Dohoku Hospital, Asahikawa, Japan

Running title: Thrombosis associated with IVC anomaly

Keywords

AMI, cerebral infarction, anomaly, deep venous thrombosis, inferior vena cava

Correspondence to Naohumi Takehara, MD

The First Department of Medicine, Asahikawa Medical College

2-1-1-1 Midorigaoka-Higashi, Asahikawa, 078-8510, Japan

Tel; +81-166-68-2442, Fax; +81-166-68-2449

E-mail; takenao@asahikawa-med.ac.jp 


\begin{abstract}
We describe a case of a 67-year-old woman with a history of cerebral infarction and pulmonary embolism that presented with chest pain. Subsequent evaluation resulted in a diagnosis of acute myocardial infarction and occult DVT, and imaging revealed a rare congenital absence of the infra-renal portion of the inferior vena cava, with lower extremity venous drainage diverted via an ascending lumbar vein. Associations between congenital absence of the inferior vena cava and thrombosis are discussed.
\end{abstract}


Introduction

Deep-venous thrombosis (DVT) may result from congenital hypercoagulable factors (eg, absence of protein $\mathrm{S} / \mathrm{C}$, lupus anticoagulant, factor $\mathrm{V}$ Leiden mutation) and/or acquired risk factors ${ }^{1-4}$. Recent reports suggest that congenital absence of the inferior vena cava (IVC) may also be an isolated risk factor for $\mathrm{DVT}^{5-8}$. Congenital absence of IVC, such as atresia or total absence, is a rare vascular anomaly that occurs during development, regression, and anastomosis of the three sets of paired veins that form the IVC 9,10 . The present study describes a case of a DVT and acute myocardial infarction (AMI) in a 67-year-old woman with congenital absence of the IVC.

Case report

A 67-year-old woman with a prior history of cerebral infarction and pulmonary embolism was hospitalized after presenting with six hours of left anterior chest pain. Electrocardiography showed ST elevation and T-wave inversion in leads $\mathrm{V}_{1}-\mathrm{V}_{4}$, and laboratory testing showed elevations in various indicators of myocardial damage, including creatine phosphokinase, creatine phosphokinase-myocardial band, aspartate transaminase, and lactate dehydrogenase. Echocardiography revealed left ventricular anterior wall motion abnormalities.

Based on these studies, a diagnosis of acute myocardial infarction (AMI) was made, and intravenous heparin and isosorbide dinitrate was instituted with subsequent 
resolution of the patient's chest pain. Physical examination failed to reveal lower extremity varices or superficial venous dilatation. Routine laboratory tests revealed moderate hyperlipidemia, and blood coagulation studies performed 14 days after hospitalization revealed elevated levels of D-dimer, thrombin-antithrombin, plasminogen activator inhibitor-1, thrombomodulin, activated von Willebrand factor, and factor VIII (>200 U/dl). However, laboratory testing was negative for lupus anti-coagulant and homocysteinemia or for deficiencies of antithrombin, plasminogen, protein S, protein $\mathrm{C}$ or factor $\mathrm{V}$. Further, the patient had no recent history of immobilization or trauma and had no familial history of thromboembolic episodes.

Coronary angiography was performed but failed to reveal significant atherosclerotic disease except for typical segmental coronary arterial narrowing in the left anterior descending artery. In contrast, venography performed via the right femoral and left dorsalis pedis veins demonstrated hypoplastic bilateral femoral veins with tapering in the intra-pelvic portion. Further, venous blood flow drained through the ascending lumbar vein connected to the femoral vein to the hepatic part of the IVC (Figure 1). Computed tomography scans of the abdomen and pelvis revealed absence of the infra-renal portion of the IVC (Figure 2), and venous blood from the lower extremities drained via the ascending lumbar vein and other collateral plexus. However, the bilateral renal veins drained into the renal portion of the IVC, and the upper venous portion of IVC drained normally into the right atrium. Thus, complete absence of IVC was limited to the infra-renal portion, affecting venous drainage from the lower extremities. 
The patient was treated with oral warfarin potassium, titrated to achieve a international normalized ration of prothrombin of 2.0-2.3, and remained free of further thrombotic events when seen in follow-up in June 2004.

\section{Discussion}

Congenital absence of the IVC, including the subsets of absence, agenesis, anomalous, and interruption of a particular segment (eg, hepatic, pre-renal, renal and infra-renal portions), is a rare vascular anomaly caused by abnormal development of the great vessels between the sixth and eighth gestational week ${ }^{9}, 10$. Absence of the IVC is frequently associated with other congenital anomalies, including polysplenia, asplenia, situs inversus, and isolated levocardia. Ninety percent of previously reported cases of congenital absence of the IVC consisted of absence of the hepatic (supra-renal) segment of IVC with azygos continuation $8,10,11$. However, the patient from the present case demonstrated absence of the infra-renal segment of IVC, and venous drainage from the lower extremities was achieved via the ascending lumbar vein to the intact hepatic and renal segment of the IVC. This type is especially rare and is estimated to comprise less than $1 \%$ of all cases of congenital absence of the $\mathrm{IVC}^{12,13}$.

AMI can rarely result from thrombosis associated with anti-phospholipids syndrome, congenital absent of protein $\mathrm{S}$ or $\mathrm{C}$, factor $\mathrm{V}$ Leiden mutations, and coronary ectasia. The patient described in the present case did not possess these abnormalities but did have evidence of multiple and recurrent thrombotic events (pulmonary embolism and 
cerebral infarction). Recently, Ruggeri et al. ${ }^{5}$ suggested that congenital absence of IVC is also an isolated risk factor for idiopathic DVT, which raises the possibility that some coagulation abnormality related to the absence of a portion of the IVC resulted in the thrombotic events in the present patient. Alternatively, the marked segmental narrowing observed in the left anterior descending artery may have resulted in thrombotic obstruction and AMI.

In summary, we described a case with a rare congenital anomaly of the absence of the infra-renal portion of the IVC that may contribute to the development of multiple and recurrent thrombotic events in this patient. 
Reference

1. Rosendaal FR. Venous thrombosis: a multicausal disease. Lancet. 1999;353:1167-73.

2. Castaman G, Ruggeri M, Tosetto A, F. R. Heterogeneity of activated protein C resistance phenotype in subjects with compound heterozygosity for HR2 haplotype and FV Leiden mutation (R506Q) in factor V gene. Thromb Haemost. $2000 ; 84: 357-8$

3. Tosetto A, Rodeghiero F, Martinelli I et al. Additional genetic risk factors for venous thromboembolism in carriers of the factor $\mathrm{V}$ Leiden mutation. $\mathrm{Br} J$ Haematol. 1998;103:871-6.

4. van Boven HH, Vandenbroucke JP, Briet E, Rosendaal FR. Gene-Gene and Gene-Enviroment interactions determine risk of thrombosis in families with inherited antithrombin deficiency. Blood. 1999;94:2590-94.

5. Ruggeri M, Tosetto A, Castaman G, Rodeghiero F. Congenital absence of the inferior vena cava: a rare risk factor for idiopathic deep-vein thrombosis. The Lancet. 2001;357:441.

6. Chee YL, Culligan DJ, Watson HG. Inferior vena cava malformation as a risk factor for deep venous thrombosis in the young. British Journal of Haematology. 2001;114:878-880.

7. Obemosterer A, Aschauer M, Schnedl W, W.Lipp R. Anomalies of the Inferior Vena Cava in Patients with Iliac Venous Thrombosis. Annals of Internal 
Medicine. 2002;136:37-41.

8. Shah NL, Shanley CJ, Prince MR, Wakwfield TW. Deep venous thrombosis complicating a congenital absence of the inferior vena cava. Surgery. $1996 ; 120: 891-896$.

9. Bass JE, Redwine MD, Kramer LA, Huynh PT, Harris JH. Spectrum of congenital anomalies of the inferior vena cava: Cross-sectional imaging findings. Radio Graphics. 2000;20:639-652.

10. Chuang VP, Mena CE, Hoskins PA. Congenital anomalies of the inferior vena cava. Review of embryogenesis and presentation of a simplified classification. $\mathrm{Br}$ J Radiol. 1974;47:206-213.

11. Fukuzawa J, Haneda T, Tobise K, Onodera S. A case report of isolated levocardia without intracardiac anomalies associated with sick sinus syndrome. Japanese Circulation Journal. 1993;57:245-250.

12. Debing E, Tielemans Y, Jolie E, Van den Brande P. Congenital absence of inferior vena cava. Eur J Vasc Surg. 1993;7:201-203.

13. Bass JE, Redwine MD, Kramer LA, Harris JH. Absence of the infrarenal inferior vena cava with preservation of the suprarenal segment as revealed by $\mathrm{CT}$ and MR venography. Am J Roentgenol. 1999;172:1610-2. 
Figure. Legend

Figure 1 Absence of the infra-renal portion of the inferior vena cava, as demonstrated by bilateral venography from the lower extremities.

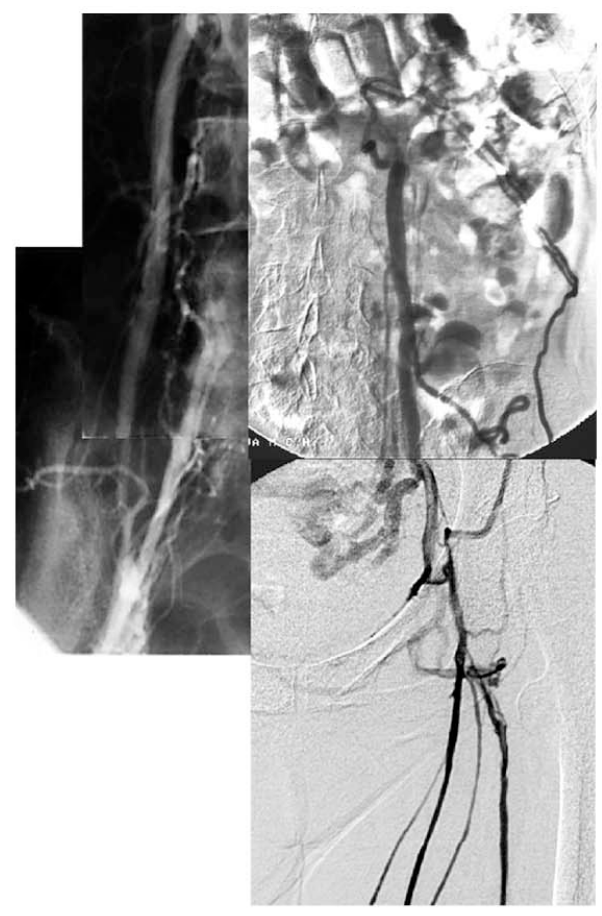

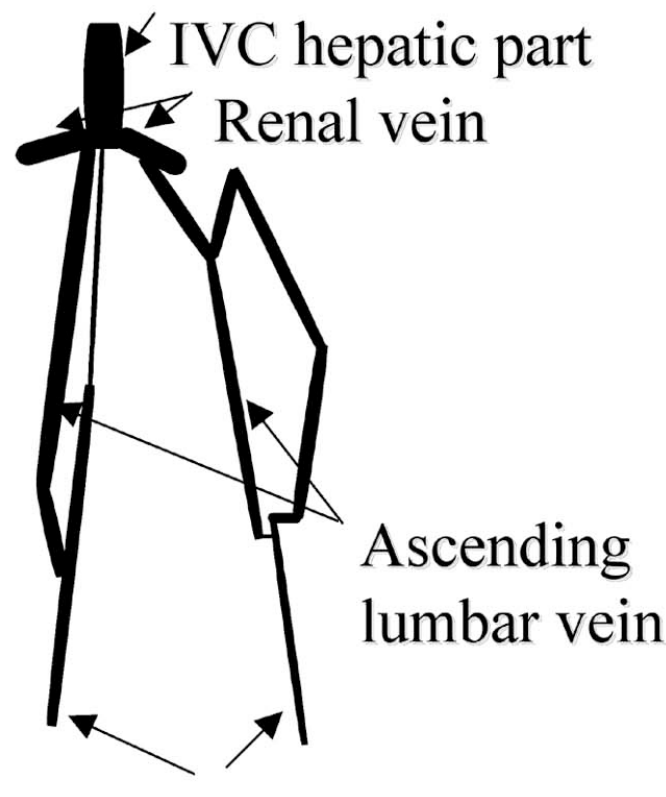

Femoral vein 
Figure 2 Computerized tomography (CT) findings. Normal appearance of the (a) hepatic portion of the inferior vena cava (IVC) and (b) renal portion of the IVC. (c) Venous drainage from the right ascending lumbar vein to the renal portion of the IVC. (d) CT imaging at the level of the pelvis demonstrated absence of the infra-renal portion of the IVC and the bilateral pelvic veins. Venous drainage was achieved via an enlarged ascending lumbar vein.

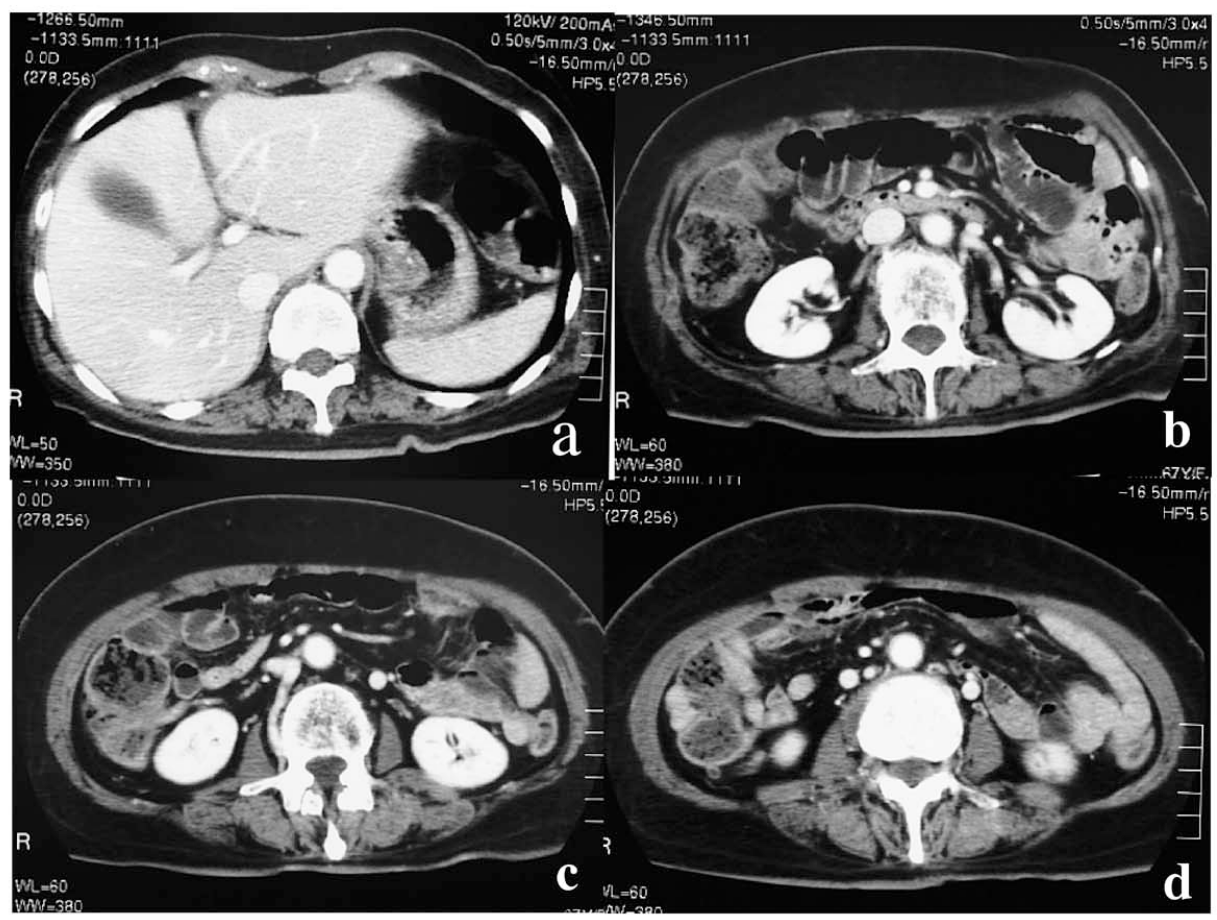

